\title{
SOBRE EL PRESENTE PRO FUTURO EN EL ESPAÑOL CONTEMPORÁNEO
}

\author{
Witold Sobczak \\ Universidad de Łódź \\ http://dx.doi.org/10.18778/8220-201-4.28
}

\section{Resumen}

En este artículo se analiza una serie de factores de índole semántico-sintáctica que condicionan el uso del presente pro futuro en el español contemporáneo. Además de destacar distintos tipos de oraciones en las que es obligatorio el empleo de la forma canta con referencia a lo posterior, se llama la atención sobre la importancia del contexto en la correcta interpretación de la referencia temporal transmitida por el presente. Por último, se comenta el uso de algunos marcadores temporales que expresan inmediatez $y$, por tanto, se refieren más bien a la simultaneidad que a la posterioridad.

Palabras clave: Presente pro futuro, posterioridad, sistema temporal castellano, tiempos verbales.

\section{1.}

\section{Introducción}

A la hora de comentar la repartición de las formas de posterioridad en castellano, en numerosas ocasiones se llama la atención sobre el presente pro futuro, denominado también presente prospectivo (Gili Gaya, 1994 [1943]: 155-156; Hernández Alonso, 1968: 30-31; Alcina/Blecua, 1991 [1975]: 793-794; Alarcos Llorach, 1994: 157; Polanco, Gras Manzano y Santiago Barriendos, 2004: 66; NGLE, 2009: $\$ 23.6$ n), cuyo empleo es común tanto en las lenguas de 
procedencia indoeuropea, p. ej., en alemán, finés, polaco, así como no-indoeuropea, p. ej., en turco (Skawiński, 2006: 112). Según la definición presentada en la NGLE (2009: § 23.6n),

el presente pro futuro se caracteriza por aludir a hechos posteriores al momento de la enunciación. Sin embargo, difiere del futuro en que -con escasas excepciones- precisa de la aparición de un complemento que aporte la información necesaria para poder situar la acción en un momento posterior de la línea temporal. El uso del presente es característico aunque no exclusivo, de los compromisos, así como de las afirmaciones rotundas, la descripción de planes, actuaciones previstas o programadas y otros sucesos venideros cuyo acaecimiento no se pone en duda.

No cabe la menor duda de que el empleo del presente pro futuro no puede considerarse una novedad desde el punto de vista diacrónicol, puesto que ya en latín era un recurso común. Como apunta Meyer-Lübke (1972 [1899]: 118, apud Meier, 1965: 72), una comparación entre el latín y las lenguas románicas llevada a cabo por este autor reveló que el presente con referencia a lo venidero, utilizado en el lenguaje popular latino, típico para la primera persona y frecuentemente acompañado por un adverbio temporal, había contribuido significativamente a la supresión del futuro sintético dabo, etc. y este proceso "parece repetirse" en la Romania actual. Gili Gaya (1962: 1) y Meier (1965: 74), a su vez, señalan que, al igual que en latín, el presente pro futuro siempre ha estado presente en las lenguas románicas, ocupando una posición ininterrumpida en la tradición latino-románica, lo cual coincide con las observaciones de Pawlik (2016: 163), según el cual dicho tiempo puede extenderse hacia el pasado o hacia el futuro con valor anticipador.

1 Curiosamente, hay lenguas que nunca han llegado a desarrollar un futuro sintético, p. ej. el hitita y siguen aprovechando el presente, a veces matizado con marcadores de tiempo, pero no necesariamente, para denotar futuridad (Pedrero, 1993: 59). 


\section{Restricciones semántico-sintácticas en el uso del presente pro futuro}

A juicio de Hernández Alonso (1968: 31), el futuro como tal es una forma vacilante e imprecisa, y por este motivo el hablante busca un sustituto preferente próximo a sí mismo, es decir, el presente, huyendo más de la noción futura que de la forma temporal de futuro. En la misma línea parecen pronunciarse Gili Gaya (1962: 2) y Pedrero (1993: 59) en cuya opinión el futuro como categoría verbal es reciente y no unitaria en las lenguas europeas, completando la oposición temporal presente/pasado. El hecho de que el empleo modal de cantará sea muy frecuente ${ }^{2}$ sugiere que tenemos que ver con una repulsa hacia la noción temporal de lo venidero y no hacia lo formalmente futuro. En realidad, la línea de intercambio es doble y compensatoria, de modo que el presente (además de la perífrasis), adquiere un valor de futuro y, en compensación, la forma de futuro sirve para denotar probabilidad en presente (Hernández Alonso, 1968: 37).

Todo ello proviene del desdoblamiento de la función significativa del futuro que encierra dos contenidos esenciales: temporalidad futura y eventualidad de acción. Como advierte Pedrero (1993: 60), en la categoría de futuro interfiere una serie de matices modales: deseo, voluntad, intención, etc., que hacen que "lo que ha de venir pueda ser deseado y trasplantado del futuro al presente" y por eso con frecuencia se recurre al presente pro futuro al expresar futuridad.

En opinión de Bravo (2008: 288-299), el tiempo en cuestión no deja de ser controvertido y no hay unanimidad en torno a la referencia temporal que le corresponde. Sea como fuere, nos parece que la descripción más compleja y transparente del valor temporal

2 Como apuntaba Gili Gaya (1962: 6) ya en la década de los sesenta, los campesinos de Castilla de aquella época evitaban cuanto podían el futuro normal, utilizándolo profusamente en sentido de probabilidad: Qué contento estará, No sé qué hora será, Estará en su casa. 
del presente pro futuro la propone Rojo (1974: 101), afirmando que la relación entre el presente prospectivo y el futuro nos permite observar que en la oposición simultaneidad/posterioridad, el término no marcado son las formas de simultaneidad. Adviértase que las formas de posterioridad siempre se refieren a lo venidero (en este caso prescindimos evidentemente de sus valores modales), mientras que las de simultaneidad pueden denotar simultaneidad o posterioridad. Este hecho está vinculado con la perspectiva temporal cero que poseen las formas de simultaneidad: son el término no marcado de todo el sistema verbal. Por tanto, si aparece en la oración algún elemento indicador de una relación $+\mathrm{V}$ (p. ej.: mañana, la próxima semana, dentro de unos días, etc.), se admite el empleo de una forma de simultaneidad a fin de referirse a una acción posterior. "En cambio, el empleo de una forma de posterioridad supone forzosamente (si no hay inclusión de valor extratemporal) referir la acción a un momento posterior al origen o a la referencia" (Rojo, 1974: 101).

Según destaca Perlin (2004: 433), la posibilidad de sustituir el futuro por el presente se ve condicionada por una serie de factores entre los cuales destacan: 1) el significado del lexema verbal; 2) el contexto; 3) el empleo de diferentes complementos circunstanciales de tiempo y 4) el tipo de acto de habla. En lo que atañe al primer factor, se trata de verbos que, utilizados en presente, suponen "haber tomado la decisión", p. ej.: Me voy de aquí, Me echan del trabajo. En general, son verbos que expresan una acción cuya ejecución depende de la voluntad, puede ser controlada y no dura demasiado tiempo. En cambio, los verbos que no cumplen estos requisitos, por ejemplo: Tengo miedo, Estudio Derecho, carentes de un contexto específico, se refieren exclusivamente al presente (Perlin, 2004: 434).

Como resaltan Gili Gaya, (1994 [1943]: 155), Alcina/Blecua (1991 [1975]: 793-794) y Moreno de Alba (1985: 34-35), el presente pro futuro aparece con frecuencia en expresiones interrogativas directas cuando uno pregunta por órdenes, decisiones que hay que dar o tomar para realizarlas después: ¿Le digo que espere un momento?, ¿Bajas en la próxima? La forma en cuestión también se admite en ciertas circunstancias en las que se ve que la acción 
va a producirse dentro de poco tiempo, p. ej.: Conchita Martínez hace el segundo servicio o Canta María Fernanda. En este caso el tiempo en cuestión suele venir acompañado de adverbios como: mañana, la semana que viene, el lunes, ya, enseguida, dentro de + cantidad de tiempo, etc.) o marcadores de modalidad epistémica como seguro, igual, fijo (Perlin, 2004: 434). Polanco, Gras Manzano y Santiago Barriendos (2004: 671), a su vez, indican que el presente en función del futuro se emplea para expresar repetición cíclica de eventos, es decir, acciones que tienen lugar en una fecha concreta, situándose en el mismo punto del eje temporal, p. ej.: Pasado mañana es miércoles. Además, el valor del futuro, aunque más inminente y con matiz de obligatoriedad, se da en el presente imperativo (Hernández Alonso, 1986: 333): Vuelves a tu casa y le pides perdón a tu hermano. Por último, el presente pro futuro aparece en ciertos actos de habla, p. ej. amenazas: Te mato, Te rompo la cabeza.

De acuerdo con lo expuesto en el artículo de Polanco, Gras Manzano y Santiago Barriendos (2004: 674), el presente prospectivo es una forma marcada, usada principalmente en situaciones muy modalizadas ${ }^{3} \mathrm{o}$ informales. Utilizar el presente en función del futuro supone una modificación de su valor temporal, de manera que este primero pasa a denotar posterioridad con respecto al origen. A pesar de que sería muy arriesgado afirmar que canta, al expresar lo venidero, se somete al mecanismo de dislocación temporal, es evidente que tenemos que ver con el fenómeno que afecta hasta cierto punto a la repartición de las formas de futuridad en el sistema verbal castellano.

A juicio de Perlin (2004: 434), en castellano hay pocas restricciones de índole semántica que impidan el empleo del presente pro futuro. Siguiendo la regla general, su uso se admite siempre que no hay duda de qué tiempo real se trata. La imposibilidad de utilizarlo se manifiesta, en primer lugar, con un verbo de sentimiento, juicio o de estado, aunque estas restricciones no tienen carácter absoluto, de modo que la frase: ¿Tú crees que si voy a Noruega allí

3 Como apunta Moreno Burgos (2014: 96), el presente se ha modalizado hasta expresar un valor de posterioridad. 
tengo frío? parece perfectamente aceptable (Perlin, 2004: 434). Sea como fuere, es obvio que el presente que expresa posterioridad requiere más esfuerzo de interpretación (para distinguir correctamente su valor prototípico) y, por eso, de su empleo deben derivarse más efectos contextuales, más información.

\section{3.}

\section{Empleo obligatorio del presente pro futuro}

En lo referente a la mencionada repartición de las formas de futuridad en castellano, cabe distinguir entre los contextos en los que el uso del presente pro futuro es obligatorio y los casos en los que el hablante al denotar lo venidero puede elegir, al menos, entre dos formas pertenecientes al mismo modo 4 . Sin lugar a dudas, el presente es indispensable en la prótasis de oraciones condicionales del primer tipo donde se admiten muy excepcionalmente la perífrasis verbal <ir $a+$ infinitivo $>$ : Si van a enseñar a leer a los niños, necesitarán libros o incluso el futuro registrado en el español popular de El Salvador y de otros países centroamericanos. En esta construcción también se rechaza actualmente el futuro de conjetura: Si acaso ella lo \{sabe $\sim{ }^{*}$ sabrá\}, a diferencia de lo que encontramos en los textos medievales (NGLE, 2009: $\$ 23.140$ ). A pesar de ello, creemos que sería erróneo eliminar totalmente las oraciones condicionales del análisis de la repartición de las formas encargadas de expresar futuridad, porque el presente puede sustituir con éxito al futuro en la apódosis de oraciones condicionales: Si ahora no comes, en una hora tienes hambre (Perlin, 2004: 434; Polanco, Gras Manzano y Santiago Barriendos, 2004: 669), lo cual

4 Nuestras consideraciones acerca de las formas utilizadas para denotar posterioridad no se refieren a los casos de la alternancia de los modos indicativo y subjuntivo que se produce, por ejemplo, en las oraciones de relativo (como en la situación que vean/verán al llegar), en algunas subordinadas adverbiales, como las concesivas (aunque lo sepan/sabrán) o en las interrogativas indirectas: No sé si te guste/gustará este platillo, etc. 
demuestra claramente la competencia entre canta y cantará. Es de subrayar que el mismo tipo de rivalidad se documenta en la proposición principal de oraciones temporales en las que se admite la forma canta: Cuando termines, salimos ${ }^{5}$.

Asimismo, al comentar el empleo obligatorio del presente en función del futuro, conviene fijarse, por lo menos, en dos locuciones adverbiales: a lo mejor e igual:

(1) A lo mejor deciden cerrarle el changarro y lo echan a la caIle. (Hayen, Calle, apud NGLE, 2009: §25.14n)

(2) Ese igual no vuelve por aquí en su vida, vete a saber. (Grandes, Aires, apud NGLE, 2009: § 25.14ñ).

Cabe destacar que las dos aprovechan casi exclusivamente el presente para denotar posterioridad con respecto al origen, aunque se registra en escasas ocasiones la presencia de la perífrasis o del futuro sintético:

(3) Si los soltamos ahora nos van a armar un rebullicio y a lo mejor vamos a tener que matarlos.

(Herrera Luque, F. (1985), En la casa del pez que escupe el agua. Caracas: Pomaire en CREA)

(4) Pero es que estudia funciones que a lo mejor no hará nunca, para ir aprendiendo.

(Fernán Gómez, F. (1982), Las bicicletas son para el verano. Madrid: Espasa Calpe en CREA)

(5) El público paraguayo no debe sentirse decepcionado porque igual vamos a clasificary antes de que finalicen las eliminatorias les vamos a dar más satisfacciones.

(Clarín (Argentina), 08/07/1997: "Incidente en el aeropuerto" en CREA)

(6) Ahí residía la dificultad: las almas jamás se exteriorizan ni ocupan un espacio determinado; en cambio, los cuerpos

5 Recordemos que el futuro no se emplea, salvo algunas excepciones, con adverbios relativos temporales en el español contemporáneo, a diferencia de lo que se admite en otras lenguas románicas y de lo que era habitual en el español de otras épocas (NGLE: 2009: $\$ 23.14 \mathrm{v})$. 
insisten en permanecer. Uno puede matar la vida que los anima, aplastarlos, triturarlos, pero igual seguirán, sustituyéndose unos a otros tenazmente.

(Gasulla, L. (1979), Culminación de Montoya, Barcelona: Destino en CREA)

Sin ir más lejos, no consideramos necesario incluir el uso de dichas locuciones en los análisis dedicados a la repartición de las formas de posterioridad del modo indicativo, puesto que casi siempre vienen seguidas por el presente para referirse a lo venidero. En otras palabras, el empleo de a lo mejor e igual con el presente no nos aporta mucha información sobre la rivalidad entre las formas que denotan posterioridad.

\section{4.}

\section{¿Simultaneidad o posterioridad?}

A pesar de que la caracterización del presente que expresa el porvenir parece bastante transparente, la distinción entre el presente usado con referencia a lo simultáneo y el presente pro futuro no deja de plantear dificultades. En nuestra opinión, para determinar si el tiempo en cuestión denota simultaneidad o posterioridad, hay que analizar detenidamente el contexto, sobre todo en los casos en los que la forma canta carece de un marcador temporal. Veamos algunos ejemplos:

(7) ¿Por qué no te tomas una pastilla?

(8) ¿Me pasas la sal?

(9) Me voy de aquí.

(10) Estudia o llamo a tus padres.

(11) O me dices qué ha pasado o no te ayudo.

Fíjese en que las oraciones presentadas supra expresan más bien lo simultáneo que lo posterior, dado que el hablante espera que su sugerencia, petición o mandato, etc. se cumplan inmediatamente. Aun así, es posible que en algún contexto la pregunta 
¿Por qué no vas al dentista? pueda denotar posterioridad si, por ejemplo, son las once de la noche y un amigo nuestro se queja de dolor de muelas. Como es bien sabido, los dentistas no suelen trabajar de noche y, por tanto, se desprende con facilidad que con este consejo el hablante se refiere al día siguiente.

La misma observación corresponde, por ejemplo, a la pregunta ¿Bajas en la próxima? que, a nuestro modo de ver, podría clasificarse como el presente o el presente pro futuro según el contexto. Si uno nos hiciera esta pregunta en el autobús que circula entre Madrid y Sevilla, y para cada dos horas, sería más natural interpretarla como vinculada a lo posterior. En cambio, al oírla en un autobús urbano, que tarda unos cinco minutos en llegar a la próxima parada, sería perfectamente posible relacionarla con lo simultáneo.

En cuanto al presente pro futuro acompañado de un complemento temporal, consideramos oportuno llamar la atención sobre los adverbios que implican semánticamente la inmediatez, p. ej. ahora mismo, enseguida, pronto, etc. y, por ello, al igual que las oraciones (7-11), se refieren más bien a la simultaneidad que a la posterioridad. He aquí algunos ejemplos:

(12) Ahora mismo subimos.

(13) Vuelvo enseguida.

(14) Acaban pronto.

\section{5. \\ Observaciones finales}

De acuerdo con lo que se ha dicho anteriormente, no existen muchas restricciones de índole semántica ni sintáctica que impidan el empleo del presente pro futuro en castellano. Sin embargo, es indispensable distinguir entre los contextos en los que su empleo es (casi) obligatorio y los en los que dicho tiempo compite con la perífrasis < ir $a+$ infinitivo $>$ y el futuro sintético. Tal distinción puede desempeñar un papel crucial en los análisis dedicados a la repartición de las formas de posterioridad, ya que permite restringir el 
material estudiado exclusivamente a los casos donde el hablante tiene la posibilidad de elegir entre, al menos, dos formas.

En lo tocante a la oposición entre el presente con referencia a lo simultáneo y el presente pro futuro, hemos visto que la serie de criterios para distinguir entre ellos no siempre resulta satisfactoria. A raíz de ello, la interpretación de la forma canta no deja de ser algo subjetiva y puede despertar polémica. Tal y como hemos señalado, parece que algunas oraciones que se relacionan a veces con el presente pro futuro: ¿Por qué no te tomas una pastilla? ¿Me pasas la sal? e implican la realización inmediata de una acción deben interpretarse más bien como el presente con referencia a lo simultáneo, a diferencia de las oraciones como: Te llamo mañana, Nos vemos en dos horas, que se refieren evidentemente a lo venidero. En cambio, la oración ¿Bajas en la próxima? puede admitir dos interpretaciones según el contexto.

\section{Referencias bibliográficas}

Alarcos Llorach, E. (1994), Gramática de la lengua española, Madrid: Espasa Calpe.

Alcina Franch, J. y Blecua, J. M. (1991 [1975]), Gramática española, Barcelona: Ariel, S.A.

Bravo, A. (2008a), La perífrasis «ir a +infinitivo» en el sistema temporal y aspectual del español, tesis doctoral inédita, [en línea] <https://www.researchgate.net/profile/Ana_Bravo5/ publication/39656884_La_perifrasis_ir_a_infinitivo_en el_sistema_temporal_y_aspectual_del_espanol/links/552bd5280cf29b22c9c1feb6/La-perifrasis-ir-a-infinitivo-en-el-sistema-temporal-y-aspectual-del-espanol.pdf $>$, [fecha de consulta: 24.01.2019].

CREA: Corpus de Referencia del Español Actual [en línea] <http:// www.rae.es/recursos/banco-de-datos/crea $>$, [fecha de consulta: 6.04.2018].

Gili Gaya, S. (1994 [1943]), Curso superior de sintaxis española, Barcelona: Bibliograf.

Gili Gaya, S. (1962), "El futuro en el lenguaje infantil”, Strenae. Estudios de filología e historia dedicados al profesor Manuel García Blanco, 16, Salamanca, 215-220. 
Hernández Alonso, C. (1968), "El futuro absoluto de indicativo", Archivum, 18, 29-39.

Hernández Alonso, C. (1986), Gramática funcional del español, Madrid: Gredos.

Meier, H. (1965), "Futuro y futuridad", R.F.E, XLVIII, 62-82.

Meyer-Lübke, W. (1972 [1899]), Grammatik der romanischen Sprachen, Hildesheim: Olms.

Moreno Burgos, J. (2014), "La expresión de la posterioridad en español”, Lenguas Modernas, 44, 81-102.

Moreno de Alba, J. G. (1985), Valores de las formas verbales en el español de México, México: Universidad Nacional Autónoma de México.

Pawlik, J. (2016), El español medieval: hacia la consolidación de un idioma, t.2. Poznań: Wydawnictwo Naukowe UAM.

Pedrero, R. (1993), "El futuro perifrástico en las lenguas europeas", Revista española de lingüística, 23, 1, 59-72.

Perlin, J. (2004), "El presente en función de futuro en polaco y en español”, Studia Romanica Posnaniensia, 31, 433-436.

Polanco, F., Gras Manzano, P. y Santiago Barriendos, M. (2004), "Presente, Ir a + infinitivo y Futuro: ¿Expresan lo mismo cuando se habla del futuro?", Actas del XV Congreso Internacional de ASELE, Sevilla, 668-674.

Real Academia Española, (2009), Nueva gramática de la lengua española, Madrid: Espasa Calpe.

Rojo, G. (1974), “La temporalidad verbal en español”, Verba, 1, 68-149.

Skawiński, J. (2006), ”Teraźniejszość w przyszłości: o użyciu czasu teraźniejszego w funkcji przyszłego", en A. Dąbrowska y A. Nowakowska (eds.), Czas - język - kultura. Język a Kultu$r a, 19$, Wrocław: Wydawnictwo Uniwersytetu Wrocławskiego, 111-114. 